\title{
An Analytic Solution of High $\beta$ Equilibrium in a Large Aspect Ratio Tokamak
}

\author{
S.C. Cowley, P.K. Kaw*, R.S. Kielly, and R.M. Kulsrud \\ Princeton Plasma Physics Lab̀oratory \\ P.O. Box 451, Princeton, New Jersey 08543
}

\section{Abstract}

An analytic solution of the high $\beta\left(\epsilon \bar{\beta}_{p} \sim \beta \eta^{2} / \epsilon \gg 1\right)$ equilibrium of a large aspect ratio tokamak is presented. Two arbitary flux functions, the pressure profile $p(\psi)$ and the saiety factor profile $q(\psi)$, specify the equilibrium. The solution splits into two asymptotic regions: the core region where $\psi$ is a function of the major radius alune and a narrow boundary layer region adjoining the conducting wall. The solutions in the two regions are asymptotically matched to each other. For monotonic pressure profiles, the Shafranov shift is equal to the minor radius. For $\beta$ much bigger than one, the sclution contains a region (in place of the magnetic axis) of zero magnetic field and constant pressure. At high $\beta$ the quantity $\beta_{i}$, which is essentially proportional to the pressure over the total current squared, is largely independent of pressure. We discuss the important ramifications of limited $\beta_{I}$ for high $\beta$ reactors. Generalizations to shaped cross sections and hollow pressure profiles are sutlined. We also consider the problem of equilibrium reconstruction in the high $\beta$ regime.

*Permanent address, Institute for Plasma Research, Gandingar Highwav Bhat, Gandingar, Gujrat, India. 1 


\section{Introduction}

There are many reasons why achieving high $\beta$ is considered desirable in tokamaks. Perhaps the simplest reason is that one wants to minimize $B^{2}$ to keep the magnet costs low and to maximize $p$ to maximize fusion power output. One would also like to achieve high pressure without supplying large volt-seconds (and therefore energy) to the transformer. This second requirement is often linked to $\beta_{I}$ which is the average pressure divided by the current squared. This link assumes that the current must be driven by the iranstormer (or possibly noninductive current drive) and therefore large $\beta_{I}$ is desirable. In this paper we show that at high $\beta$ (specifically $\epsilon \vec{\beta}_{p} \sim \beta q^{2} / \epsilon \gg 1$ see definition of $\vec{\beta}_{p}$ below) $\epsilon \beta_{I}$ is of order one and essentially independant of pressure and $\epsilon$. This, however, does not mein that one needs a large transformer to obtain high beta. If a high beta equulibrium is obtained from a low beta equilibrium via a flux conserving sequence, no volt seconds need be supplied. Sustaining the current at high beta also may not require a large transformer if the temperature is high and the resistance correspondingly low.

Equilibrium at high $\beta$ (strictly speaking $\beta \sim O(1)$ ) is not well understood. Much of our intuitioc about tokamak equilibrium comes from low $\beta$, large aspect ratio tokamak equilibrium. The analytic solutions for large aspect ratio, high $\beta$, tokamak equilibrium given in this paper are qualitatively different from low $\beta$ solutions. We hope the solutions presented here will extend our understanding of tokamak equilibrium to high $\beta$. We have been able to investigate arbitrarily high values of $\beta$ sesulting in equilibria that are qualitatively different 
from those found previously in numerical studies. To solve for an equilibrium, we must specify two functions of the poloidal flux function $\psi$. We choose to specify the safety factor $q(\psi)$ and the pressure profile $p(\psi)$. The total poloidal flux $\psi_{\max }$ is also specified. Previous high $\beta$ solutions s, $^{1,2}$ assume specific pressure and toroidal field profiles. Our solutions are, however, valid for arbitary $q$ and $p$ profiles. The specification of $q(\psi)$ is motivated by the flux conserving tokamak (FCT) concept. ${ }^{1}$ In the FCT, a high $\beta$ plasma is obtained by heating a low $\beta$ plasma on a time scale shorter than the resistive time. This means that $q(\psi)$ is conserved as $p(\psi)$ jncreases during the heating. It is reasonable to specify the pressure profile since it can be measured experimentally.

In our analysis we make two assumptions: that $\epsilon \ll 1$ and that $\epsilon \bar{\beta}_{p} \gg 1$ where $\epsilon \equiv a / R_{0}$ and $\bar{\beta}_{p} \equiv\left(a^{2} R_{0}^{2} / \psi_{\text {max }}^{2}\right) \mu_{0} p_{\text {max }}$. Here $a$ is the mean minor radius, $R_{0}$ is the mean major radius and $p_{m a s}$ is the maximum value of the pressure. For the majority of the paper we assume the equilibrium is bounded by a circular conducting wall. The solution is easily generalized to shaped boundaries (see Section VIb). We contrast our analysis with previous large aspect ratio $€$ quilibrium expansions. ${ }^{3.4}$ These expansions are valid if $\epsilon \ll 1$ and $\epsilon \bar{\beta}_{p} \ll 1$. These equilibria have circular flux surfaces and a small Shafranov shift. On the other hand, oui high $\beta$ equilibrium solution has D-shaped flux surfaces and a large Shafranov shift of approximately the minor radius. Note that the regions of validity for these two solutions do rot overlap.

Simple qualitative physical considerations allow us to deduce the two most striking 
features of high beta large aspect ratio equilibria. These features are: (i) the existence of a boundary layer; and (ii) the fact that outside the boundary layer, the poloidal flux is a function solely of the major radius. In the poloidal plane the forces on the plasma may be divided into three parts: the pressure force $-\nabla p$, the force due to the toroidal field $-\nabla B_{T}^{2} / 2-B_{T}^{2} \nabla R / R_{\mathrm{r}}$ and the force due to the poloidal field $-\nabla B_{\mathrm{p}}^{2} / 2+\mathrm{B}_{\mathrm{p}} \cdot \nabla \mathrm{B}_{\mathrm{p}}$. Note that from $\mathbf{B} \cdot \nabla \mathbf{p}=0$ we deduce that $p=p(\psi)$ and from $\mathbf{J} \cdot \nabla \mathrm{p}=0$ we deduce that $B_{T}=F(\psi) / R$. For $\beta$ of order one, $p / B_{T}^{2} \sim O(1)$, the pressure and toroidal forces are comparable. For $q \sim O(1)$ [see Eq. (3)] we find that nominally $B_{p} / B_{T} \sim O(\varepsilon / q)$. Thus the force due to the poloidal field is nominally smaller than the pressure and toroidal field forces by a factor of order $\epsilon^{2}$.

The balance of toroidal field forces and pressure forces yields $R^{2} \nabla p \sim \nabla F^{2}$ which, since $p=p(\psi)$ and $F=F(\psi)$, implies $\psi=\psi(R)$. This balance does not give confinement as is well known - because the flux surfaces are not closed. A flux surface that is closed has an unbalanced force of order $\epsilon \nabla p$ due to the lack of balance between $R^{2} \nabla p$ and $\nabla F^{2}$. This unbalanced force can only be balanced by forces due to the poloidal field. The only way to increase the poloidal field force for a given poloidal flux is to squash the flux into a smaller volume. If one squashes the flux into a smaller volume (henceforth called the boundary layer) of width $\delta$ in the poloidal plane, the poloidal field is increased from its nominal value $\left(B_{p} / B_{T} \sim O(\epsilon / q)\right)$ by a factor $a / \delta$. One can determine $\delta$ by balancing the unbalanced part of the pressure and toroidal field forces against the enhanced poloidal field force, $-\nabla(\epsilon p) \sim \nabla\left(a^{2} \epsilon^{2} B_{T}^{2} /\left(\delta^{2} q^{2}\right)\right)$. Therefore $\delta / a \sim O\left(\left(\epsilon / \beta q^{2}\right)^{1 / 2}\right) \sim O\left(\left(\epsilon \tilde{\beta}_{p}\right)^{-1 / 2}\right)$. 
It is obvious that a given flux surface $\operatorname{can}^{\prime} t$ be entirely in a boundary layer of width $\delta$ otherwise $q \sim O(\delta)$. Physically it is clear that the poloidal field cannot be large over the whole surface if the rotational transform is to be finite. It is also obvious that a given flux surface cannot be entirely outside the boundary layer since it has to violate $\psi=\psi(R)$ somwhere in order to close. We show below that, for monotonic pressure profiles, the boundary layer must follow the fixed conducting wall. Thus, every flux surface must have a straight section where $\psi=\psi(R)$ and a section in the boundary layer following the conducting wall. In the circular boundary case, the surfaces are circles with a straight section (roughly D shaped). These qualitative conclusions are born out by the asymptotic analysis of Section IV.

In Section II of this paper, the initial equations are presented. Based on assumptions of finite $q(\psi)$ and high $\beta$, we scale the variables appropriately and rewrite these equations in terms of the small parameter $\epsilon$ (Section III). By performing an asymptotic expansion in $\epsilon$, we solve for the poloidal flux function $\dot{\psi}(x, z)$ in the core in Section IVa., and in the boundary layer in Section IVb. The core solution and the boundary layer solution correspond to the straight and curved parts of the D-shaped fiux surfaces, respectively. It is worth remarking that the core solution does not depend on the boundary layer solution and it can be computed by a simple one-dimensional integration. In Section IVc. it js shown that a region of perfect diamagretism appears in the center of the plasma when $\beta$ is greater than a critical value of order one. The main results are given in Eqs (12) and Eq. (13) for the core solution and Eq. (20) for the boundary layer. Using our solutions, we investigate 
the various poloidal betas in Section $V$. We find that $\epsilon \beta_{I} \rightarrow \pi^{2} / 16$ as $\beta \rightarrow \infty$ and that $\epsilon \beta_{I}$ is essentially independent of pressure and $\epsilon$ for $\beta \leq 1$. This contradicts the conciusion of Clarke and Sigmar..$^{5}$ In Appendix A we investigate the behavior near the magnetic axis. We show that the solution contains a third boundary layer at the magnetic axis. The difficulties associated with the magnetic axis affects a small fraction of order $\epsilon^{3 / 4}$ of the total flux and can be ignored for most considerations. The problem of experimentally deducing $q(\psi)$ in these equilibria is considered in Appendix B. We show that $q$ can be inferred from a measurement of the pressure along the midplane (the line $\theta=0$ in Fig.1). We also show that $q(\psi)$ cannot be inferred from a measurement of the poloidal field on the boundary.

\section{Initial Equations}

For an axisymmetric system the ideal Magnetohydrodynamic (MHD) equilibrium equations reduce to the Grad-Shafranov equation:

$$
\left[R \frac{\partial}{\partial R}\left(\frac{1}{R} \frac{\partial}{\partial R}\right)+\frac{\partial^{2}}{\partial z^{2}}\right] \psi=-\mu_{0} R^{2} \frac{d p}{d \psi}-F \frac{d F}{d \psi}
$$

The magnetic field $\mathbf{B}$ is given by

$$
\mathbf{B}=\frac{1}{R} \nabla \psi \times \mathrm{e}_{\phi}+\frac{F}{R} \mathrm{e}_{\phi}
$$

In this paper, we solve the Grad-Shafranov equation for a tokamak with major ridius $R_{0}$ and minor radius $a$ as shown in Fig. 1. The boundary is a perfectly conducting shell with a circular poloidal cross-section. As boundary conditions, we find it convenient to 
take $\psi=0$ at the wall and to assume $\psi=\psi_{\max }$ is given at the magnetic axis. Thus, $\psi$ increases inwards contrary to most conventions.

The specified free functions are the pressure profile $p(\psi)$ and the safety factor $q(\psi)$. To close the equations, we need an equation relating $q(\psi)$ and $F(\psi)$. It is given by

$$
q(\psi)=\frac{F(\psi)}{2 \pi} \oint \frac{d l_{\triangleright}}{R|\nabla \psi|}
$$

where $d l_{p}$ is a poloidal line element. The integration is performed along poloidal projections of surfaces of constant $\psi$. We take $F$ and $q$ to be positive.

\section{Dimensionless Equations}

To simplify the analysis we introduce the following dimensionless notation:

$$
\bar{z}=z / a, R=R_{0}(1+\epsilon \bar{x}), \epsilon=a / R_{0}, \bar{\psi}=\psi / \dot{\psi}_{\max }, \overline{d l}_{p}=d l_{p} / a
$$

The barred variables are dimensionless. The inverse aspect ratio $\epsilon$ is assumed small and is our expanzion parameter.

We assume that the safety factor, $q(\psi)$, is finite and that we have a high $\beta$ equilibrium. That is, $q(\psi) \sim O(1)$ and $\mu_{0} p_{f}^{\prime} B_{\phi}^{2} \sim O(1)$. Combining these assumptions with the definitions of the toroidal and poloidal components of the magnetic field, we are lead to the scalings for $F$ and $p$. These, in turn, lead to the introduction of the following dimensionless variables:

$$
\bar{F}=\frac{a^{2}}{R_{0} \psi_{\max }} F, \quad \bar{p}=\frac{\mu_{0} a^{q}}{\psi_{\max }^{2}} p .
$$


We define an average poloidal beta:

$$
\bar{\beta}_{p} \equiv \frac{\mu_{0} p_{\max } a^{2} R_{0}^{2}}{\psi_{\max }^{2}} \equiv \bar{p}_{\max } \epsilon^{-2}
$$

so that $\varepsilon \bar{\beta}_{p} \sim O\left(\varepsilon^{-1} \beta q^{-2}\right)$.

In the scaled variables, the Grad-Shafranov equation reduces to the dimensionless equation

$$
-\epsilon^{2}\left[(1+\epsilon \bar{x}) \frac{\partial}{\partial \bar{x}} \frac{1}{(1+\epsilon \bar{x})} \frac{\partial}{\partial \bar{x}}+\frac{\partial^{2}}{\partial \bar{z}^{2}}\right] \bar{\psi}=(1+\epsilon \bar{x})^{2} \frac{d \bar{p}}{d \bar{\psi}}+\bar{F} \frac{d \tilde{F}}{d \tilde{\psi}}
$$

Eq. (3) becomes

$$
\tilde{q}(\bar{\psi})=\frac{\bar{F}(\bar{\psi})}{2 \pi} \oint \frac{d \bar{l}_{p}}{(1+\epsilon \bar{x})|\bar{\nabla} \bar{\psi}|}
$$

where $\bar{\nabla}$ operates in the barred variables. The scaled boundary conditions are: $\vec{\psi}=0$ on $\bar{x}^{2}+\tilde{z}^{2}=1 ;$ and $\bar{\psi}=1$ on the magnetic axis. Equations $(6)$ and $(7)$ are, of course, exact - in our subsequent asymptotic theory we treat $\bar{p}, \bar{q}, \bar{F}$ and $\bar{\psi}$ to be order one in an asymptotic expansion in the small parameter $\epsilon$. Let us be very specific about the ordering of beta. We choose $\tilde{p} \sim O\left(\epsilon^{2} \bar{\beta}_{p}\right) \sim O(\beta) \sim O(1)$. This is a maximal ordering in the sense that it allows the toroidal field forces and the pressure forces to compete. In Section IVb we show that the boundary layer is narrower than the minor radius if $\bar{p} \gg \epsilon$, or equivalently $\epsilon \bar{\beta}_{p} \gg 1$. In fact $\epsilon \bar{\beta}_{p} \gg 1$ and $\epsilon \ll 1$ are sufficient conditions for the validity of our expansion.

From this point onward, hopefully without confusion, we will drop the bar notation, except where explicitly stated.

\section{Solution}


We immediately recognize the form of Eq. (6) to be that of a singular perturbation problem. As we shall see, it is in fact a boundary layer problem.

\section{a. The Core Solution.}

First we examine the solution obtained by treating $\psi$ as finitely varying in $x$ and $z$. This is referred to as the "outer solution" in boundary layer theory. However, since we expect the boundary layer to be outside this region at $x^{2}+y^{2} \sim 1$, we refer to this solution as the core solution to avoid confusion. To find an approximate solution to Eq. (6), we expand $\psi$ and $G \equiv F F^{\prime}$ using the small parameter $\epsilon: \psi=\psi_{0}+\epsilon \psi_{1}+\cdots$ and $G=G_{0}+\epsilon G_{1}+\cdots$. To $O(1)$ we have

$$
p^{\prime}\left(\psi_{0}\right)+G_{0}\left(\psi_{0}\right)=0
$$

Substituting $G_{0}=F_{0} F_{0}^{\prime}$ into Eq. (S) and integrating we get

$$
p\left(\psi_{0}\right)+\frac{1}{2} F_{0}^{2}\left(\psi_{0}\right)=C
$$

where $C$ is an undetermined constant. This equilibrium equation corresponds to the straight tokamak approximation where there is only a toroidal magnetic field. To $O(\epsilon)$, Eq. (6) is

$$
2 x p^{\prime}\left(\psi_{0}\right)+G_{1}\left(\psi_{0}\right)=0
$$

By dividing each term by $p^{\prime}$ and then differentiating with respect to $z$, we find that $\psi_{0}(x, z)=\psi_{0}(x)$. As we anticipated, the solution without poloidal field is one in which $\psi$ is a function of major radius alone. By looking at Eq. (6) order by order, it can be shown that $\psi(x, z)=\psi(x)$ to all orders in $\epsilon$. 
This solution to the Grad-Shafranov equation implies that the poloidal projections of the flux surfaces are vertical lines. However, we demand that the solution consist of nested surfaces. Furthermore, for these flux surfaces to satisfy the boundary condition of $\psi=0$ on the wall, a rapid spatial variation in $\psi$ is necessary. These expectations suggest the existence of a boundary layer near the conducting wall and that the surfaces have the shape illustrated in Fig. 2. Sinse $|\nabla \psi| \gg 1$ (in fact we expect $\nabla \psi \sim O\left(c^{-1 / 2}\right)$ ) in the boundary layer, the contribution from this region to the integral in Eq. (7) will be negligible to lowest order. Therefore, we can approximate Eq. (7) by

$$
\boldsymbol{q}\left(\psi_{0}\right)=\frac{ \pm F_{0}\left(\psi_{0}\right)}{2 \pi} \frac{\ell(x)}{d \psi_{0} / d x}
$$

Here, $\ell(x)=2 \sqrt{1-x^{2}}$ is the vertical line length in scaled variabies as shown in Fig. 1. Combining Eqs. (9) and (11) and integrating we get

$$
\begin{aligned}
2 \pi \int_{D}^{\psi_{0}} \frac{q\left(\tilde{\psi}_{0}\right)}{\sqrt{2\left(C-p\left(\tilde{\psi}_{0}\right)\right)}} d \tilde{\psi}_{0} & =\int_{-1}^{x} \ell(\tilde{x}) d \tilde{x} \\
& =\pi / 2+x \sqrt{1-x^{2}}+\sin ^{-1} x .
\end{aligned}
$$

Once the consiants of integration $C$ and $D$ are found, equation (12) gives an implicit relation between $\psi_{0}$ and $x$. We show below that $D$ is in fact zero and $C$ is given by Eq. (13). The sign is selected to make the left hand side of Eq.(12) positive.

Formally, one would determine $C$ and $D$ by matching to the boundary layer. However, we can deduce $C$ and $D$ without a detailed solution of the boundary layer. We shall, however, use some properties of the boundary layer that are formally derived in Section IVb. First, note that no flux surface can lie entirely in the boundary layer, i.e. some part 
of the surface must lie in the core, since from Eq. $(7),|\nabla \psi|$ must be finite for some finite distance along the surface to give a finite $q$. Secondly, since Eq.(10) has a unique solution for $x$, flux surfaces pass through the core region only once. Anticipating Eq.(19), we note that the boundary layer decays exponentially into the core only if $p^{t} /(d \psi / d x)$ is positive in the core. Here $d \psi / d x$ is given by Eq.(11).

The placement of the boundary layers and the magnetic axis are dependent on the sign of $p^{\prime}$. Let us first consider monotonically increasing $p(\psi)\left(p^{\prime}>0\right)$. More complicated $p(\psi)$ are discussed in Section VI. Along the line $z=0, \psi$ must rise from zero at $x=-1$ to $\psi=1$ at the magnetic axis, and then decrease to $\psi=0$ at $x=1$. From the above considerations, we find that the region with $d \psi / d x$ positive must be in the core, and the region with $d \psi / d x$ negative must be in the boundary layer. Since the negative $d \psi / d x$ region has a width of order $\epsilon^{1 / 2}$, the width of the boundary layer, the boundary layer locates the magnetic axis at $x \sim 1-O\left(\epsilon^{1 / 2}\right)$. There is no boundary layer at $x=-1$ so consequently $D=0$. Note the plus sign in Eq.(12) is therefore appropriate. In order for the magnetic axis to have zero thickness and the solutions to match, the core must locate $\psi=1$ at $x \sim 1-O\left(\epsilon^{1 / 2}\right)$. To lowest order this implies

$$
\int_{0}^{1} \frac{q\left(\tilde{\psi}_{0}\right)}{\sqrt{C-p\left(\tilde{\psi}_{0}\right)}} d \tilde{\psi}_{0}=\frac{1}{\sqrt{2}}
$$

For moderate and low $p(\psi)$ Eq.(13) determines C. The integral on the left handside of Eq. (13) is a monotonically decreasing function of $\mathrm{C}$. The maximum value of the left hand side of Eq.(13) is attained when $C=p(1)$. if this maximum value is less than $1 / \sqrt{2}$, then 
no solution to Eq.(13) exists! (Note that $C$ must be real and larger than $p(1)$ since $F$ is real.) This contradiction arises at lrege $p(\psi)$. A qualitative change in the solution takes place for these cases, sprcificaliy the magnetic axis occupies a finite region throughout which $p=p(1)$ and $\mathrm{B}=0$. We call this region of perfect diamagnetism the "hole". This solutions is discussed in Section IVc. In the following section, Section IVb, we assume that Eq.(13) can be satisfed. We emphasize that the core solution can be computed via Eqs. (12) and(12) without computing the boundary layer solution.

When $E \ll 1$ (and $\epsilon \bar{\beta}_{p} \gg 1$ ) we can simplify the core solution. In this limit, Eq. (9) yields $F_{c}^{2}=2 C$ (i.e. $F_{0}$ is independent of $\dot{\psi}_{0}$ ) and $p\left(\psi_{0}\right)$ can be ignored in Eqq. $(1 \Sigma)$ and (13). Thus, when $\epsilon / q^{2} \ll \beta \ll 1$ the toroidal field is a constanc to lowest order and $\psi_{0}(\tau)$ for $s\left(\psi_{n}\right)$ il is independent of pressure.

As an example, letı us find $\psi_{0}(x)$ from Eqs. (12) and (13) for the case $q=q_{0}=$ constant and $p=p_{0}^{\prime} \psi$. We obtain

$$
\psi=\frac{A}{\pi}\left(1+\frac{\beta_{0}}{16}\right)-\frac{A^{2}}{\pi^{2}} \frac{\beta_{0}}{16}
$$

where $A=\pi / 2+x \sqrt{1-x^{2}}+\sin ^{-1} x$ and $\beta_{0}=p_{0}^{\prime} / q_{0}^{2}$. Equation (14) is valid for $0<\beta_{0}<16$, when $\beta_{0}>16$ we have a "hole" solution. We plot this $\psi_{0}(x)$ in Fig. 3 for various values of $\beta_{0}$. A iso included in Fig. 3 is 2 "hole" case with $\beta_{0}=64$.

\section{b. The Bound ary Layer Solution.}

For the boundiry leyer solution we work in the polar coordinates $(r, \theta)$ with the or:gin 
situated at the center of the poluidal cross section. In these coordinates, Eq. (6) becomes

$$
\begin{array}{r}
-\epsilon^{2}\left[\frac{1}{r} \frac{\partial}{\partial r}\left(r \frac{\partial}{\partial r}\right)+\frac{1}{r^{2}} \frac{\partial^{2}}{\partial \theta^{2}}-\frac{\epsilon}{1+\epsilon r \cos \theta}\left(\cos \theta \frac{\partial}{\partial r}-\frac{\sin \theta}{r} \frac{\partial}{\partial \theta}\right)\right] \psi \\
=(1+\epsilon r \cos \theta)^{2} \frac{d p}{d \psi}+F \frac{d F}{d \psi} .
\end{array}
$$

We look for solutions where $\partial / \partial r \sim O\left(\epsilon^{-1 / 2}\right)$. This ordering ensures that, to lowest order, Eq. (15) reduces to Eq. (8). To introduce this scaling, we define the boundary layer thickness variable $t$ such that $r=1-\epsilon^{1 / 2} t$. Keeping terms up to $O\left(\epsilon^{3 / 2}\right)$, equation (15) becomes

$$
\left(-\epsilon \frac{\partial^{2}}{\partial i^{2}}+\epsilon^{3 / 2} \frac{\partial}{\partial t}\right) \cdot \psi=\left[1+\epsilon\left(1-\epsilon^{1 / 2} t\right) \cos \theta\right]^{2} \frac{d p}{d \psi}+F \frac{d F}{d \psi} .
$$

Hecause $1 / 2$ powers of $\epsilon$ appear in Eq. (16), we expand $\psi$ and $G \equiv F F^{\prime}$ as follows:

$$
\begin{gathered}
\psi=\psi_{0}+\epsilon^{1 / 2} \psi_{1 / 2}+\epsilon \psi_{1}+\epsilon^{3 / 2} \psi_{3 / 2}+\cdots \\
G=G_{0}+\epsilon^{1 / 2} G_{1 / 2}+\epsilon G_{1}+\epsilon^{3 / 2} G_{3 / 2}+\cdots
\end{gathered}
$$

We substitute these expansions into Eq. (16) and equate the terms order by order. As required, the $O(1)$ equation reproduces Eq. (8). The $O\left(\epsilon^{1 / 2}\right)$ equation implies that $G_{1 / 2}=0$, which is consistent with the expansion in $\mathrm{Eq} .(10)$. For the $O(c)$ equation we get

$$
-\frac{\partial^{2} \psi_{0}}{\partial t^{2}}=2 \cos \theta \frac{d p}{d \psi_{0}}+G_{1}\left(\psi_{0}\right)
$$

Note that $\theta$ does not appear in any derivatives in Eq. (17), and thus may be treated as a parameter. Substituting $G_{1}\left(\psi_{0}\right)$ from Eq. (10) into Eq. (i7), we get

$$
\frac{\partial^{2} \psi_{0}}{\partial t^{2}}=2\left[x\left(\psi_{0}\right)-\cos \theta\right] \frac{d p}{d \psi_{0}} \equiv-\frac{d V}{d \psi_{0}}
$$


where $x\left(\psi_{0}\right)$ is given implicitly in Eq. (12). Multiplying Eq. (18) by $\partial \psi_{0} / \partial t$ and integrating we get

$$
\frac{1}{2}\left(\frac{\partial \psi_{0}}{\partial t}\right)^{2}=2 \int_{\psi_{\text {sort }}(\cos \theta)}^{\psi_{0}} d \psi^{\prime}\left(x\left(\psi^{\prime}\right)-\cos \theta\right) \frac{d p}{d \psi^{\prime}}=-V\left(\psi_{0}, \theta\right)
$$

Where $\psi_{\text {core }}(\cos \theta)$ is the core solution, given in Eq. (12), with $\psi_{0}(x)$ evaluated at $x=\cos \theta$. Note that when $\psi=\psi_{\text {core }}(\cos \theta), x(\psi)=\cos \theta$. We have chosen the boundary condition that $\partial \psi / \partial t \rightarrow 0$ as $\psi \rightarrow \psi_{\text {core }}(\cos \theta)$, so that the boundary layer solution matches to the core solution. One may easily show that as $\psi \rightarrow \psi_{\operatorname{core}}(\cos \theta)$, Eq. (18) has two solutions: one that converges to $\psi_{\text {core }}$ and one which exponentially diverges. By choosing the lower limit of integration in Eq. (19) as $\psi_{\text {core }}(\cos \theta)$, we have selected the converging solution. We use the notation $V(\psi, \theta)$ in Eq. (18) to emphasise a merhanical analogy :where $V$ represents the potential energy of a particle, $\psi$ its position, and time. $V$ is zero with zero slope at $\psi=\psi_{\text {core }}$ and is a monotonically increasing function of $\psi$ for $\psi<\psi_{\text {core. }}$. Thius the "particle" with zero total energy (potential plus kinetic energy) can be thought of as ascending a hill with just enough energy to reach the summit at $\psi=\psi_{\text {core. }}$. However it takes an infinite amount of time to get there. Equation. (19) can be integrated to yield,

$$
t=\int_{0}^{\psi_{0}} \frac{d \tilde{\psi}_{0}}{\sqrt{-2 V\left(\tilde{\psi}_{0}, \theta\right)}}
$$

where we have used the boundary condition that when $t=0$ (the wall) $\psi_{0}=0$.

We have assumed that the thickness of the boundary layer is negligible in constructing the core solution - we therefore need to verify that it is indeed narrow. To investigate 
the thickness we look at the asymptotic behavior of Eq. (20) as $\psi \rightarrow \psi_{\text {core. }}$ Since the integral in Eq. (20) is dominated by the region where $\psi_{0} \simeq \psi_{\text {core }}$, we can simply expand the denominator in the integrand to obtain

$$
t \sim \frac{-\log \left(\psi_{\text {core }}-\psi\right)}{\sqrt{2 x^{\prime}\left(\psi_{\text {core }}\right) p^{\prime}\left(\psi_{\text {core }}\right)}} .
$$

Thus the boundary layer solution approaches the core solution exponentially as $t$ goes to infinity. Noting that $r=1-\sqrt{\epsilon} t$, we find the scale length for the boundary layer thickness to be $\sqrt{\epsilon /\left(p^{\prime} x^{\prime}\right)} \sim \sqrt{\epsilon / p}$. Using the scalings Eqs (4) and (5), we find the thickness to be $\sim\left(\epsilon \bar{\beta}_{p}\right)^{-1 / 2}$. Therefore, our analysis is valid if and only if $\epsilon \bar{\beta}_{p} \gg 1$ and $\epsilon \ll 1$. Equations (19) and (20) give the boundary layer solution (to lowest order in $\epsilon$ ) and complete the full solution, with Eqs. (12) and (13), for $x\left(\psi_{0}\right)$.

\section{c. The Hole Solution}

As mentioned in Section IVa, a central region of perfect diamagnetism, the hole, can appear if $p(\psi)$ is large. Physically, such a region arises because $p+B_{\phi}^{2} / 2 \simeq$ constant and therefore, to balance a large pressure change, a large $B_{\phi}$ field is needed. However, to maintain a finite toroidal flux the large $B_{\phi}$ field is forced to occupy a smaller region.

Considering the $p^{\prime}>0$ case only, and arguing as we did in Section $\Gamma \mathrm{a}$, we conclude that on the line $z=0$, the $d \psi / d x$ positive region is in the core and the $d \psi / d x$ negative region is in the boundary layer. The position of the magnetic axis predicted by the bourdary layer is at a position $x \sim 1-O\left(\varepsilon^{1 / 2}\right)$. However, when $\mathrm{Eq}$. (13) has no solution the maximum $x$ at which the core can place the magnetic axis is, with $C=p(1)$, at $x=x_{h}$ with $x_{h}$ given 
by

$$
2 \pi \int_{0}^{1} \frac{q\left(\psi_{0}\right)}{\sqrt{2\left(p(1)-p\left(\psi_{0}\right)\right)}} d \psi_{0}=\pi / 2+x_{h} \sqrt{1-x_{h}^{2}}+\sin ^{-1} x_{h} .
$$

Note that $x_{h}$ decreases as $p(1)$ increases (for fixed $p(\psi) / p(1)$ ), and since $x_{h}$ is less than one, the magnetic axis must occupy a finite region (the hole). Throughout this hole, $\psi=1$ therefore the hole contains no poloidal field. We imagine that we have obtained this solution via a flux conserving sequence starting from a low $\beta$ finite $q$ aquilibrium without the hole. At a critical pressure $x_{h}=1$ the solution has no hole, but the toroidal field and the poloidal field are zero at the magnetic axis. Above this critical pressure the hole opens up. iv f flux surface can have poloidal field without toroidal field and since there is no poloidal fieid in the hole, there cannot be any toroidal field in the hole. Inside the hole $\nabla p=\mathbf{J} \times \mathbf{B}=0$. Thus, force halance is assured automatically. Note, however, that the Grad Shafranov equation is inappropriate in this region, as it is derived from $\nabla p=\mathrm{J} \times \mathbf{B}$ by assuming that $\nabla \psi$ is nonzero.

Across the edge of the hole $p+B^{2} / 2$ must be continuous. Hence, since $p$ is continuous and $B^{2}$ is zero in the hole, $B^{2}=0$ just outside the hole. From Eq. $(9)$, with $F(1)=0$, we find $C=p(1)$. Equation (11) then implies that $d \psi / d x=0$ in the core at the edge of the hole. Consequently, the hole bounds the core region at $x=x_{h}$. The boundary layer adjacent to the hole (where $\theta<\cos ^{-1} x_{h}$ ) has a thickness $t_{h}$. Noting that at $t=t_{h}, \dot{\psi}=1$ and $\partial \psi / \partial t=0$ for $\theta<\cos ^{-1} x_{h}$ we obtain 


$$
t_{h}(\theta)=\int_{0}^{1} \frac{d \psi_{0}}{\sqrt{2\left(-V\left(\psi_{0}, \theta\right)\right)}}
$$

Where $\psi_{\text {corc }}(\cos \theta)=1$ in the definition of $V$, Eq. (19). For $\theta>\cos ^{-1} x_{h}$ the boundary layer is the same as in Section IVb. The hole is therefore a D-shaped region and in the limit $p \rightarrow \infty$ the hole engulfs the whole cross section. In Fig. 4 we illustrate a typical "hole" equilibrium.

For the simple case $q=$ constant and $p=p_{0}^{\prime} \psi$ treated in section IVb. we obtain in the "hole" limit the core solution $\psi=A(x) \sqrt{\beta_{0}} /(2 \pi)-A(x)^{2} \beta_{0} /(4 \pi)^{2}$. This expression is valid when $\beta_{0}>16$ atherwise Eq. (14) holds. In Fig. 3 we plot $\psi(x)$ for $\beta_{0}=64$. Note that $x_{h}$ is 0 in this case.

\section{Investigation of Various Betas}

Various betas are used to characterize equilibria. Here we discuss three. The quantities $\beta_{I}, \beta_{p}$ and $\beta_{\phi}$ are defined as follows:

$$
\beta_{I}=\frac{8 \pi \int \mu_{0} p r d r c^{\prime} \theta}{\left(\mu_{0} I_{\phi}\right)^{2}}
$$

and

$$
\begin{aligned}
& \beta_{p}=2 \mu_{0} \frac{\int p d V}{\int B_{p}^{2} d^{\nabla}} \\
& \beta_{T}=2 \mu_{0} \frac{\int p d^{2} V}{\int B_{\phi}^{2} d V}
\end{aligned}
$$

where $I_{\phi}$ is the total plasma current in the toroidal direction. We have also defined $\bar{\beta}_{p}=$ $\left(a^{2} R_{0}^{2} / \psi_{\max }^{2}\right) \mu_{0} p_{\max }$ in Eq. (5). These quantities can be measurod experimentally, and are 
therefore useful for comparison with theory. Note that only at low pressure is $\bar{\beta}_{\mathrm{p}}$ the same order as $\beta_{p}$ and $\beta_{l}$.

To investigate $\beta_{l}$, we calculate $\mu_{0} I_{\phi}$ using Ampère's Law. That is

$$
\mu_{0} I_{\phi}=\oint \mathbf{B} \cdot d \mathbf{l}=\frac{\psi_{\mathrm{ma}=}}{R_{0}} \int_{0}^{2 \pi}\left|\frac{\partial \bar{\psi}}{\partial \bar{r}}\right| d \theta
$$

where the integral is around the outermost flux surface. Both scaled and unscaled variables enter into Eq. (27), and therefore we have reinstated the bars on the scaled variables. From Eq. (27),

$$
\begin{aligned}
\left|\frac{\partial \bar{\psi}_{0}}{\partial \bar{r}}\right|_{r=1} & =\frac{2}{\sqrt{\epsilon}}\left[\int_{0}^{\psi_{\operatorname{cor} \theta}(\theta)} d \bar{\psi}[\cos \theta-x(\bar{\psi})] \frac{d \bar{p}}{d \bar{\psi}}\right]^{1 / 2} \\
& =\frac{2}{\sqrt{\epsilon}}\left[\int_{-1}^{\cos \theta} \bar{p}(x) d x\right]^{1 / 2}
\end{aligned}
$$

where $\bar{p}(x) \equiv \bar{p}\left(\psi_{c c r c}(x)\right)$. To obtain the second expression, we have integrated by parts and assumed that $\breve{p}(0)=0$. Note that the current is carried almost entirely by the boundary layer. The numerator of $\beta_{I}$ can be calculated using Eq. (4):

$$
\mu_{0} 8 \pi \int p r d r d \theta=8 \pi \frac{\psi_{\max }^{2}}{a^{2}} \int_{-1}^{1} \bar{p}(x) \ell(x) d x
$$

where $\ell(x)=2\left(1-x^{2}\right)^{1 / 2}$. In Eq. (29) we keep only the core contributions to the integra! since the boundary layer is narrow.

Cembining Eqs. (24), (27), (28) and (29), we get

$$
\epsilon \beta_{I}=\frac{\frac{\pi}{2}\left(\int_{-1}^{1} \bar{p}(x) \ell(x) d x\right)}{\left(\int_{-1}^{1} \frac{d x}{\ell(x)}\left(\int_{-1}^{x} \bar{p}\left(x^{\prime}\right) d x^{\prime}\right)^{1 / 2}\right)^{2}}
$$


This formula is valid only if there is no diamagaetic hole. In the limit $\epsilon^{2} \bar{\beta}_{p} \gg 1$ the hole takes up most of the sross section, $x(\psi) \sim x_{h} \sim-1$, and for $x>x_{h},(\partial \psi / \partial r)_{r=1} \sim$ $(2 / \sqrt{\epsilon})(x+1)^{1 / 2} p_{\max }^{1 / 2}$. Computing $\beta_{I}$ we obtain

$$
\lim _{22 \hat{\beta}_{\mathrm{p}} \rightarrow \infty} \epsilon \beta_{t} \rightarrow \frac{\pi^{2}}{16}
$$

This result is independent of the functional form of $p(\psi)$.

Let us consider the behaviour of $\beta_{l}$ as pressure and $\epsilon$ are varied. This behaviour depends on what is held fixed, as pressure or $\epsilon$ varies. In the large hole limit, when $\beta \rightarrow \infty, \epsilon \beta_{I} \rightarrow \pi^{2} / 16$, as was shown above. Let us therefore concentrate on the case without a hole. First note that if we set $\vec{p}(x)=\alpha f(x)$ in Eq. $(30), \epsilon \beta_{J}$ is independent of $\alpha$. Thus, $\epsilon \beta_{l}$ remains constant if the pressure is raised, keeping the profile in $x$ fixed. If, however, one keeps $p(\psi) / p_{\max }, \epsilon, \psi_{\max }$ and $q(\psi)$ fired and raises $p_{\max }, \epsilon \beta_{f}$ does change. In the limit $\beta \ll 1$, however, $\psi_{\text {core }}(x)$ is independent of pressure (see the end of section IVa). Thus when $\beta$ is in the range $\epsilon / q^{2} \ll \beta \ll 1, p(x) / p_{\max }$ is fixed (and therefore $\beta_{I}$ constant) if $p(\psi) / p_{\max }$ is held constant as $\beta$ is raised. Lowering $\epsilon$ (it is easiest to think of letting $R_{D} \rightarrow \infty$ ) keeping everything else fixed, lecves $\epsilon \beta_{I}$ unchanged and raises $\epsilon \bar{\beta}_{p}$. One can make $\epsilon \beta_{I}$ arbitrarily large by making $\bar{p}(x)$ arbitrarily localised zibout $x=1$. These solutions are singular and may be considered unphysical. None of the above limits agree vith Clarke and Sigmar's ${ }^{5}$ claim that $\epsilon \beta_{I} \sim O\left(\left(\epsilon \bar{\beta}_{p}\right)^{1 / 3}\right)$ for large $\epsilon \bar{\beta}_{p}$. Clarke and Sigmar ${ }^{5}$ made the unjustified assumption that the fux surfaces would remain circular, and they did not recognize the need for the existence of the hole for $\epsilon^{2} \bar{\beta}_{p} \gg 1$. Not surprisingly, their 
conclusions about the behavior of $\beta_{l}$ are erroneous.

For the large hole limit $\left(\varepsilon^{2} \bar{\beta}_{p} \gg 1\right), \beta_{p}$ and $\beta_{T}$ reduce to simple expressions,

$$
\begin{aligned}
& \epsilon \beta_{p}=\frac{\pi}{\sqrt{32 \epsilon}} \frac{\bar{p}_{\max }}{\int_{0}^{l}\left(\bar{p}_{\max }-\bar{p}(\psi)\right)^{1 / 2} d \psi}, \\
& \beta_{T}=\frac{\bar{p}_{\max }}{2 \sqrt{2} \int_{0}^{1}\left(\bar{p}_{\max }-\bar{p}(\psi)\right)^{1 / 2} q d \psi} .
\end{aligned}
$$

The current profile is often specified in place of $q(\psi)$ in mumerical solutions of the GradShafranov equation. The toroidal current $I(\psi)$ enclosed inside a given flux surface $\psi$ is given by,

$$
\mu_{0} I(\bar{\psi})=\frac{4 \psi_{\max }}{R_{0} \epsilon^{1 / 2}} \int_{x(\bar{\psi})}^{1} \frac{d x}{\left(1-x^{2}\right)^{1 / 2}}\left[\int_{\bar{\psi}}^{\psi_{\text {core }}(x)}\left(x-x\left(\psi^{\prime}\right)\right) \frac{d \bar{p}}{d \psi^{\prime}} d \psi^{\prime}\right]^{1 / 2}
$$

Eq. (34) is correct with and without a hole, with the rule $\psi_{\text {rore }}(x)=1$ for $x>x_{h}$.

VI. Generalizations.

In this section we discuss three generalizations of the solutions. These have been omitted from Section IV in the interest of clarity.

\section{a. Hollow and non-monotonic pressure profiles.}

We note from Eq. (18) that in order for the boundary layer solution to match to the core solution $(x=x(\psi))$, we need

$$
\left(\frac{d p}{d \psi}\right)\left(\frac{d x}{d \psi}\right)>0
$$

where $d x / d \psi$ denotes the derivative of the function $x=x(\psi)$. When $p^{\prime}<0$ everywhere, a hollow pressure profile, Eq. (35) indicates that the magnetic axis is on the edge of the 
boundary layer at $x \sim-1$, an inward Shafranov shift. The core solutiou is then given by

$$
\begin{aligned}
\sqrt{2} \pi \int_{0}^{\psi_{0}} \frac{q\left(\psi^{\prime}\right) d \psi^{\prime}}{\left(C-p\left(\psi^{\prime}\right)\right)^{1 / 2}} & =\int_{x}^{1} \ell(\tilde{x}) d \tilde{x} \\
& =\pi / 2-x \sqrt{1-x^{2}}-\sin ^{-1} x .
\end{aligned}
$$

C is determined as before by Eq. (13). The boundary layer solution is unchanged. Clearly this case has $p>0$ at the plasma edge doesn't yield confilement.

The non-monotonic pressure profile with a single maxima is complicated, since some surfaces have $d \psi / d x$ positive in the core and some negative. The only possible configuration, given that all surfaces must pass once, and only once, through the core region, is one in which there is an inte:nal boundary layer at some $x=x_{A}$, and the magnetic axis is at $x \sim x_{A}$. Since this is not a configuration of practical interest and the complicaticns are formidable, we omit further discussion if its properties.

\section{b. Non circular conducting walls.}

A relatively trivial generalization of Section IV allows the introduction of arbitrary shaped conducting walls. Of course the voluine must still have a large average aspect ratio.

The core solution can be obtained from Eq. (12) with $\ell(x)$ replaced by the distance from the top to the bottom of the vacuum vessel at constant major radius. Note that, in the general case, the right hand side of Eq. (12) is the area in the poloidal plane bounded by the vacuum vessel and the vertical line $x=$ constant. Complications can arise when the vertical line ( $x=$ constant) intersects the vacum vessel more than twice. This would occur, for instance, in the "Bean shaped" configuration. ${ }^{6}$ The reader may verify that in this case 
of muitiple vertical segments, $\ell(x)$ is replaced by the sum of the lengths of all the segments.

As before, the boundary layer follows the conducting wall. The boundary layer solution is most easily solved in the coordinates $v$ and $u$ where $v$ is the distance along the wall and $\epsilon^{1 / 2} u$ is the perpendicular distance from the conducting wall. Note these coordinates are well defined near the wall but not necessarily everywhere. On the wall $x=H(v)$, a known function. Equations. (18), (19) and (20) are replaced with identical eqations with the replacement of $t$ by $u$ and $\cos \theta$ by $H(v)$. In a volume with multiple vertical segments there are clearly multiple boundary layer segments.

\section{c. Current Free Plasma Halo.}

The solution we've given has considerable current density at the conducting wall. This is unphysical, as the edge of the plasma is cold and highly resistive. In this section we demonstrate how a halo of currentless pressureless plasma can be constructed around one of our solutions. Consider a solution constructed as in Section IV. Let us remove the outermost flux surfaces, taking away a fraction $\delta$ (where $\delta \ll 1$ ) of the poloidal flux. Now we have an equilibrium with a boundary shape defined by the last flux surface, $\psi=\delta$. Clearly the boundary has a circular segment which bounds the boundary layer and a straight segment which is part of the core solution, see Fig. 5. We now surround the solution with a currentless plasma and demand that no surface current exist at the interface between the old solution and new halo. Therefore we make the toroidal field of the currentless plasma equal to $F(\delta) / R$. The poloidal field is made continuous by setting the jump in $\nabla \psi$ across 
the inturface to be zero.

To lowest order in $\delta$ we plare the conducting wall, $\psi=0$, a perpendicular listance $d=\delta /|\nabla \psi|_{\psi=\delta}$ outside the surface $\psi=\delta$, see Fig. 5. Note that $d$ varies considerably with poloidal angle. In the region bounded by the boundary layer, $|\theta|<\theta_{0}$ in Fig. 5 , $d \sim O\left(\delta \epsilon^{1 / 2}\right)$. The region $|\theta|>\theta_{0}$ where the currentless halo adjoins the core solution has $d \sim O\left(\delta^{2 / 3}\right)$. Note that the new conducting wall is not quite circular. The current in our halo is to lowest order in $\delta$, zero. Higher order solutions come from solving $\nabla^{2} \psi=0$ in this region with the boundary condition that $\nabla \psi$ is continuous across the $\psi=\delta$ surface. The conducting wall is placed at the $\psi=0$ surface whatever shape that surface takes. There is no separatrix to all orders in $\delta$.

\section{Conclusions.}

In this paper we present a new method for finding the high beta equilibrium of a large aspect ratio tokamak. The flux surfaces in this equilibrium have a characteristic shape. Each surface has a straight section (the core solution) where $\psi$ is a function of the major radius and a section which is confined to a boundary layer at the wall of width $\left(\epsilon \bar{\beta}_{p}\right)^{1 / 2}$. In Eqs. (12) and (13) of Section IVa we constucted the core solution for arbitrary pressure ( $p$ ) and safety factor (q) profiles. The boundary layer solution is given in Eq. (20) of Section IVb. When the pressure is very large, beta of order one, a new type of solution occurs. This solution possesses a field free "hole" replacing the magnetic axis, and is described in Section IVc. In Section IV we assumed a circular conducting wall. The generalization to 
a shaped boundary is given in Section VI. A small proportion of the poloidal flux of order $\epsilon^{3 / 4}$ is contained in a further boundary layer at the magnetic axis (in the no hole case). The magnetic axis bounda:y layer is treated in Appendix A. In Appendix B we show how the equilibrium, including the q profile, can be reconstructed from a measurement of $p$ along the line $\theta=0$. We also show that it is impossible to deduce the q frofile from external measurements of the magnetic field.

Analytic equilibria have many obvious advantages over numerical equilibria. In fact, high beta equilibria are notoriousiy difficult to compute, perhaps our solution can help such computations. At present, codes do not operate at betas large enough to find the "hole" solutions. The solution we present has several deficiencies. First, it is an asymptutic expansion in $\epsilon$ and $\left(\epsilon \bar{\beta}_{p}\right)^{-1}$ and, therefore, it is only valid at large aspect ratio and large $\epsilon \bar{\beta}_{p}$. Second, it is a fixed boundary solution, which is not realistic for most tokamaks. The conducting wall could in principle be replaced with a complex system of coils which mimic the currents flowing in the wall. Finally, our solutions have large current densities at the wall. It was shown in Section VIc that a current free region can be added between the current carrying plasma and the wall.

Four betas are defined in this paper: $\bar{\beta}_{p}$ defined in Eq. (5) which is of order the poloidal beta of the core region; $\beta_{I}$ defined in $\mathrm{Eq} .(24) ; \beta_{p}$ the average beta poloianl beta defined in Eq. (25); and $\beta_{T}$ the toroidal beta defined in Eq. (26). $\beta_{1}$ is often used in characterising an equilibrium perhaps because it is easily measured. Clarke and Sigmar claim that $\beta_{I}$ 
increases like $p^{1 / 3}$ for high betas. We show in Section $V$ that this claim is false; $\beta_{I}$ is roughly independent of pressure for $\beta_{T} \ll 1$ and $\beta_{\tau} \sim \pi^{2} / 16$ when $\beta_{T} \gg 1$. At low beta a large valu.; of $\beta_{I}$ is considered desirable because we want high pressures but low current. Current must be driven either inductively or by radio frequency current drive. Thus a limit on $\beta_{t}$ might be considered unfortunate. However, consider heating the plasma (faster than the resistive time) to high pressure. The current will increase without any applied locp voltage (or current drive) keeping $\beta_{l}$ roughly constant. In heating the plasma, the poloidal field energy increases (by a factor of order $a / \delta \sim \sqrt{\epsilon \bar{\beta}_{p}}$ ). This energy is supplied by the heating source not by the transformes. Now consider raising the temperature, $T$, in a flux conserving sequence, keeping the density constart. The plasma resistivity draps like $T^{-3 / 2}$ and the current channel (the boundary layer) narrows like $\delta \propto T^{-1 / 2}$. Therefore the resistance drops like $T^{-1}$. Keeping $\beta_{J}$ constant implies that the current risss like $T^{1 / 2}$. Thus, the loop voltage trops like $T^{-1 / 2}$. The volt-second requirement to sustain the current does fall with increasing beta (for fixed density and toroidal field).

In this paper we have considered high beta equilibria - the stability of these equilibria is not discussed. We hope to address some of the stability issues in the future. One should note that the $s-\alpha$ model $^{7}$ used to study balloning modes at high prescure is a lacal equilibrium constructed about a flux surface that is assumed to be a circle on which $|\nabla \psi|$ is a constant. In this paper we have shown that the only circular surfiace in a high beta large aspect ratio equilibrium is the wall. However, $|\nabla \psi|$ is not constant on the wall. In fact, at $\theta=\pi,|\nabla \psi|=0$ and at $\theta=3,|\nabla \psi| \sim O\left(\varepsilon^{-1 / 2}\right)$. Obviously the $s-\alpha$ model vill 
not give the correct ballooning stability boundaries of our equilibria. We do not know if the qualitative picture of ballooning stability given by the $s-\alpha$ model $r$, zains correct in our hight beta equilibria.

\section{Acknowledgments}

It is a pleasure to acknowledge the computational help of Ernie Valeo. R.M.K. wishes to acknowledge a useful discussion (some years ago) on large q equilibria with Martin Kruskal. This work was supported by DUE Contract No. DE-ACQ2-76-CH0-36i3. 


\section{A.ppendix A: Magnetic Axis Solutions}

The solution near $\theta=0$ (the magnetic axis region) is not correctly described by the procedure outlined in the text. A new internal boundary layer is needed to describe the region around the magnetic axis. This region, which we refer to as the magnetic axis region, separates the core and the boundary layer solutions. Note that we continue to iefer to the region adjoining the wall as the boundary layer. The new region is illustrated in Fig. 6. (This figure is not drawn to scale). The corrections described in this appendix are negligible except for an order $\varepsilon^{3 / 4}$ amount of the poloidal flux even at $\theta=0$. Thus, the solution given in the text describes all but an asymptotically small fraction of the flux surfaces.

There are essentially two reasons why the solution given in the text breaks down at near the magnetic axis. First, the evaluation of q given in Section IVa becomes incorrect when $1-\psi \sim O\left(\epsilon^{3 / 4}\right)$ where the width of the boundary layer significantly shortens the length, $\ell(x)$, of the surface in the core region. When $1-\psi \sim O(\varepsilon)$ the surfaces are no longer $\mathrm{D}$ shaped and the surfaces lie entirely in the magnetic axis region. In this region the equations are a two dimensional integro-differential system which cannot be solved easily. We have obtained an approximate solution which makes $q$ correct at the magnetic axis $(\psi=1)$ and correct for $1-\psi \gg \epsilon$. The second reason for a modified treatment is that the matching between the core and boundary layer solutions breaks down because in the region $1-\psi \sim O\left(\epsilon^{3 / 4}\right)$ the $\epsilon^{1 / 2} t$ term on the right hand side of $\mathrm{Eq}$. (16) becomes important,

The function $G_{1}(\psi)$ must be modified so that $q(\psi)$ remains correct in this region $\left[G_{0}\right.$ is 
still given correctly by Eq. (8)]. Let us first consider the behavior of the solution with $G_{1}(\psi)$ given by Eq. (10). From Eqs. (12) and (13) for $\Delta \psi=1-\psi \ll 1$ (and $\Delta x=1-x \ll 1$ ) we obtain

$$
\Delta x(\Delta \psi)=(\alpha \Delta \psi)^{2 / 3}
$$

where $\alpha=q(1)(3 / 4) \pi(C-p(1))^{-1 / 2}$. At the point $\theta=0$,and nowhere else, the integral in Eq. (20) converges as $\psi \rightarrow \psi_{\text {care }}=1$. We define the width of the boundary layer in $t, t_{0}$, by the expression

$$
t_{0}=\int_{0}^{\tau} \frac{d \tilde{\psi}_{0}}{\sqrt{-2 V\left(\tilde{\psi}_{0}, 0\right)}} .
$$

Inserting Eq. (37) into Eq. (18) [or Eq. (21)] we find the typical distance, $\epsilon^{1 / 2} \Delta t$, for the boundary layer to exponentially decay into the core (for small $\theta$ ) to be order $\theta^{1 / 3} \epsilon^{1 / 2}$. Thus, while the boundary iayer has a finite width in $t$ (for small $\theta$ ), it has a much sharper edge, i.e. $\Delta t \ll \dot{i}_{0}$.

Now consider a $D$ shaped surface where the tip of the $D$ is at $\theta_{0} \sim O\left(\epsilon^{1 / 4}\right)$. The $q$ of this surface is wrong if we take $G_{1}$ to be given in the manner described in Section IVa since we didn't take into account the width of the boundary layer in computing the length of the surface in the core, $\ell(x)$. If we assume, correctly it turns out, that the width of the boundary layer is not significantly altered by the corrections at the magnetic axis, we may replace $\ell(x)$ by $\vec{\ell}(x)=2 \sqrt{\left(1-\epsilon^{1 / 2} t_{0}\right)-x}$ in Eq. (12). On the straight part of the D, Eq. (37) yields, $|\nabla \psi| \sim d \psi / d x \sim(\Delta x)^{1 / 2} \sim O\left(\epsilon^{1 / 4}\right)$. On the curved part of the D, however, $|\nabla \psi| \sim O\left(\Delta \psi / \theta_{0}^{1 / 2} \epsilon^{1 / 2}\right) \sim O\left(\epsilon^{1 / 4} / \theta_{0}^{1 / 2}\right)$. Thus the irtegral defining $q$, Eq. ( 7$)$, is still dominated by the straight section. We show below that with this modified $\ell(x), \bar{\ell}(x)$, we 
obtain $G_{1}(\psi)=-2 x(\psi) p^{\prime}(\psi) \sim 2 p^{\prime}(1)\left[(\alpha \Delta \psi)^{2 / 3}-1+\epsilon^{1 / 2} t_{0}\right]$ for $\Delta \psi \ll 1$.

The width of the $D$, i.e. the distance from the curved to the straight part at $\theta=0$, is of order $\theta_{T}^{2}$ where $\theta_{T}$ is the angle of the tip of the $\mathrm{D}$. When this width is comparable to the exponentiation length of the boundary layer, $\theta_{T}^{1 / 2} \epsilon^{1 / 2}$ the surfaces are clearly no longer D shaped. Thus when $\theta_{T} \sim O\left(\epsilon^{1 / 3}\right)$ the whole procedure for obtaining the correct $q$ profile, which relies on a separation of the surface into a straight core region and a boundary layer region, breaks down. One can estimate that $\Delta \psi \sim O(\epsilon)$ when $\theta_{T} \sim O\left(\epsilon^{1 / 3}\right)$ by using the $\Delta x(\psi)$ as modified above by the width of the boundary layer. The problem in this region becomes one of finding $G_{1}(\psi)$ so that $q(\psi) \sim q(1)$. We show below that for $\Delta \psi \ll 1 G_{1}(\psi)$ takes the form

$$
G_{1}(\psi) \sim 2 p^{\prime}(1)\left[-1+\left(\alpha\left(\Delta \dot{\psi}+\Delta \psi_{0}\right)\right)^{2 / 3}+\epsilon^{1 / 2} t_{0}+\epsilon^{2 / 3}\left(2 \alpha p^{\prime}(1)\right)^{-2 / 3} H(\Lambda \psi / \epsilon)\right]
$$

where $\Delta \psi_{0}$ is a constant of order $\epsilon$ and the unknown function $H(u)$ must satisfy $H(u) \ll$ $u^{2 / 3}$ for $u \rightarrow \infty$. $H$ is determined by an integro differential system. We have not been able to solve for $H$ so that $q$ is correct everywhere. However, by relacing $H$ by a constant of order one, we have made $q$ on axis correct and $q$, of course, is correct for $\Delta \psi \gg \epsilon$.

The mathematical development of the solution around the magnetic axis is complicated. Let us therefore summarize the various regions that appear in the analysis (see Fig. 6). When

$$
\pi \geq|\theta| \gg \epsilon^{1 / 4}
$$

the core and boundary layer regions are correctly described by the solutions given by 
Eqs. (12) and (20) of Section IV of the text. In the region

$$
\pi \gg|\theta| \gg \epsilon^{1 / 3}, \quad|\theta| \sim O\left(\epsilon^{1 / 4}\right)
$$

the core and boundary layer solutions are modified by the width of the boundary layer but no new boundary layer is needed. The regions of validity in t when $\theta$ obeys Eq. (41) are, for the core solution,

$$
t-t_{0} \gg|\theta|^{1 / 2}, t>t_{0}
$$

and for the boundary layer,

$$
0 \leq t \leq t_{1}, 0<t_{1}-t_{0} \ll 1
$$

Note that the core and boundary layer solutions overlap in the region $1 \gg t-t_{0} \gg|\theta|^{1 / 2}$.

The region

$$
|\theta| \sim O\left(\epsilon^{1 / 3}\right) \ll \epsilon^{1 / 4}
$$

contains three regions to be matched asymptotically. These regions sre, for the core region

$$
t-t_{0} \gg \epsilon^{1 / 6}
$$

for the magnetic axis region

$$
\left|t-t_{0}\right| \sim O\left(\epsilon^{1 / 6}\right) \ll 1
$$

and for the boundary layer region

$$
0 \leq t<t_{1}, t_{0}-t_{1} \gg \epsilon^{1 / 6}
$$


Clearly the core and axis regions overlap in the region $1 \gg t-t_{0} \gg \epsilon^{1 / 6}$ and the axis and boundary layer regions overlap in the region $1 \gg t_{0}-t \gg \epsilon^{1 / 6}$.

\section{Modified core solution}

Let us begin the mathematical development by considering the effect of the boundary layer width on the core solution. Substituting the modified form of $\ell(x), \bar{\ell}(x)$ into Eq. (11) we obtain for $\Delta x \ll 1$

$$
\pi-\frac{4 \sqrt{2}}{3}\left(\Delta x-\epsilon^{1 / 2} t_{0}\right)^{3 / 2} \sim 2 \pi \int_{0}^{1} \frac{q(\psi) d \psi}{(2 C-2 p(\psi))^{1 / 2}}-\frac{2 \pi q(1) \Delta \psi}{(2 C-2 p(1))^{1 / 2}}
$$

where $\Delta \psi=1-\psi$ and $\Delta x=1-x$. Since we are expecting the magnetic axis to be close to $\Delta x=\epsilon^{1 / 2} t_{0}$ we may determine $C$ to lowest order via Eq. (13). (We call this value $C_{0}$ ). While it is sufficient to use $C=C_{0}$ in the core region when $\Delta x \gg \epsilon^{1 / 2}$, a more careful evaluation is required for $\Delta x \sim O\left(\epsilon^{1 / 2}\right)$. Let us define $\Delta \psi_{0}$ by the equation,

$$
2 \pi \int_{0}^{1} \frac{q(\psi) d \psi}{(2 C-2 p(\psi))^{1 / 2}}-\pi=\frac{2 \pi q(1)}{\left(2 C_{0}-2 p(1)\right)^{1 / 2}} \Delta \psi_{0}
$$

Also let $\alpha \equiv q(1)(3 / 4) \pi\left(C_{0}-p(1)\right)^{-1 / 2}$. Then Eq. (48) yields

$$
\left(\Delta x-\epsilon^{1 / 2} t_{0}\right)=\left[\alpha\left(\Delta \psi+\Delta \psi_{0}\right)\right]^{2 / 3}
$$

where $\Delta \psi_{0}$ which is of order $\epsilon$ is a parameter to be evaluated in the matching.

\section{Modified boundary layer solution}

Now consider the effect of the boundary layer width on the boundary layer solutions.From Eqs. (10) and (16) we obtain for $\theta \ll 1$,

$$
\frac{d^{2} \psi}{d t^{2}}=-2 \frac{d p}{d \psi}\left[-\Delta x(\psi)+\epsilon^{1 / 2} t_{0}+\frac{\theta^{2}}{2}\right]+2\left(t-t_{\mathrm{G}}\right) \epsilon^{1 / 2} \frac{d p}{d \psi} .
$$


The $\epsilon^{1 / 2} t$ term which is revritten as $\epsilon^{1 / 2} t_{0}+\epsilon^{1 / 2}\left(t-t_{0}\right)$, has been retained since it is comparable to $\Delta x(\psi)$ and $\theta^{2} / 2$ in the matching region. The part of this term proportional to $t-t_{0}$ is dropped because when $\Delta x(\psi)$ is finite it is unimportant, and (we show apostiori) when $\Delta x(\psi) \sim \theta^{2} \sim O\left(\epsilon^{1 / 2}\right)$ we find $\epsilon^{1 / 2}\left(t-t_{0}\right) \sim O\left(\theta^{1 / 2} \epsilon^{1 / 2}\right)$. Equation (51) is integrated in the manner given in Section IVb. Note that the $\epsilon^{1 / 2} t_{0}$ term in Eq. (51) cancels with the $\epsilon^{1 / 2} t_{0}$ correction to $\Delta x(\psi)$ given in $\mathrm{Eq}$. (50) when $\Delta \psi \ll 1$. After some algebra the following limiting expressions for the boundary layer solutions are obtained, for $\theta^{3} \ll \Delta \bar{\psi} \ll 1$

$$
\Delta \psi \sim \alpha^{2}\left(t-t_{0}\right)^{6}\left(\frac{p^{\prime}}{15}\right)^{3}
$$

and for $\left(2^{3 / 2} \alpha \Delta \psi / \theta^{3}\right)-1 \ll 1$

$$
\Delta \psi \sim \frac{\theta^{3}}{2^{3 / 2} \theta}+A \exp -\frac{\left(t-t_{0}\right) B}{|\theta|^{1 / 2}}
$$

where $B=\sqrt{\alpha p^{\prime}} 2^{5 / 4} / \sqrt{3}$ and $A \sim O(1)$. Thus as before, without the boundary layer width corrections, the boundary layer solution approaches the core solution, Eq. (.50), exponentially with a width in $t$ of order $|\theta|^{1 / 2}$. Note that the boundary layer has a wirlth (in $t$ ) to plus corrections of order $|\theta|^{2 / 2}$. The assumption used to derive the core solution is therefore justified when the exponential decay length $|\theta|^{1 / 2} \epsilon^{1 / 2}$ is short compared to the width of the D-shaped surface, $\theta^{2}$ (i.e. where $\theta \gg \epsilon^{1 / 3}$ ). Thus, by modifying the core solution, $\mathrm{Ec}_{1}$. (50), and the boundary layer solution, Eq. (51), we have extended the solution to the region $\epsilon^{1 / 3} \ll \theta \sim O\left(\epsilon^{1 / 4}\right)$.

\section{Magnetic Axis Region}

Let us now consider the region $\theta \sim O\left(\epsilon^{1 / 3}\right)$ and $\Delta \psi \sim O(\epsilon)$, the magnetic axis region. 
The term proportional to $t-t_{0}$ in Eq. (51) must be included in this region. The separation of the core and boundary layer regions is impossible in this limit. Therefore $G_{2}(\psi)$ cannot be determined from the core solution. Let us introduce the following scalings,

$$
\begin{gathered}
\left(\Delta \psi_{0}+\Delta \psi\right)=\frac{\epsilon W}{2 p^{\prime}(1) \alpha^{2}} \\
t-t_{0}=\frac{\epsilon^{1 / 6} s}{\left(\alpha 2 p^{\prime}(1)\right)^{2 / 3}}
\end{gathered}
$$

and

$$
\theta=\frac{\sqrt{2} \epsilon^{1 / 3}}{\left(\alpha 2 p^{\prime}(1)\right)^{1 / 3}} \overline{\hat{\theta}}
$$

With these normalisations and the form of $G_{1}(\psi)$ given in Eq. (39) and Eq. (51) we obtain,

$$
\frac{d^{2} W}{d s^{2}}=W^{2 / 3}-s-\bar{\theta}^{2}+H(W) \text {. }
$$

Equation (57) correctly describes the solution when $W \ll \epsilon^{-1}$. In order to match the core solution we require the boundary condition,

$$
W \rightarrow\left(\bar{\theta}^{2}+s\right)^{3 / 2}
$$

as $s \rightarrow \infty$. When $s \rightarrow-\infty$ the solution of Eq. (57) must match limit of the boundary layer solution given in Eq. (52),

$$
W \sim \frac{1}{(30)^{3}}(s)^{6}
$$

From $5 q .(7)$ and $q \sim q(1)$ in this region we obtain,

$$
\frac{4}{3}=\oint \frac{d \bar{\theta}}{\left|\frac{d W}{d s}\right|}
$$


where the integration is taken over the $W=$ conetant contour. The mathematical problem as then to find a function $H(W)$ so that $W$, the solution of Eq. (57) obeying the boundary conditions Eqqs. (58) and (59), satisfies the constraint Eq. (60). The problem [finding $H(W)]$ is independent of the specific $q(\psi)$ and $p(\psi)$, thus it can be solved once and for all. Unfortunately we have not been able to solve this problem in a rigourous manner. We have, however, obtained a solution by replacing $H(W)$ by a constant $s_{0}$ and choosing $s_{0}$ so that $q$ is correct on the magnetic axis. The $q$ condition on axis becomes,

$$
3 \pi=\left[\frac{\partial W}{\partial \theta^{2}} \frac{\partial^{2} W}{\partial s^{2}}\right]^{1 / 2}
$$

where the derivatives are evaluated at $W=W_{\min }$, the magnetic axis. We find numerically that for $s_{0}=10.1$ and $W_{\min }=11.5$. Note that $\epsilon W_{\min }=2 p^{\prime} \alpha^{2} \Delta \psi_{0}$, thus $\Delta \psi_{0}$ is order $\epsilon$ as has been assumed. In Fig. $\neg$ we plot the approximate solution. Although the plot does not extend to the asymptotic limits in either direction, the computation was performed over a range extending to the asymptotic limits. Demanding that $q=q(1)$ over this region may not be the most physically relevant constraint; perhaps a more realistic presrciption would be to demand a level of smoothness to the current profile, since short scale variations in the current are rapidly destroyed by resistive diffusion. 


\section{Appendix B: Equilibrium Reconstruction.}

There are very few direct measurements of $q(\psi)$ in experiments. This is unfortunate since q plays a cruicial role in stability, especially in sawtooth oscilations. The most common way to infer $q$ is via equilibrium reconstruction. ${ }^{8}$ In this technique (in its most basic form) the Grad-Shafranov equation is solved for many $q(\psi)$ and $p(\psi)$ profiles. These solutions are then compared with the experimentally measured $p(\mathbf{r})$ and the magnetic field at the boundary of the plasma. The $g(\psi)$ profile of the solution which most nearly fits the measurements is then inferred to be the experimental $q$ profile.

In this appendix we consider the problem of dedıcing $q(\psi)$ for our equilibrium. We show that the $q$ profile cannot be inferred from measuremeriss of the magnetic field on the boundary. However $q$ can, be inferred from a knowledge of the pressure profile along the line $\theta=0$, (core and boundary layer profiles are needed) and the toroidal field at the boundary. We will first assume a circular boundary then generalise to shaped boundaries.

First let us consider the information obtained from the magnetic field at the boundary. From $E_{q 1} .(4)$ and $(9)$ and the condition $p(0)=0$ we obtain,

$$
C=\frac{a^{4} B_{T}^{2}(0)}{\psi_{\max }^{2}}
$$

where $B_{T}(0)$ is the toroidal field at the wall in unscaled units. The $O(\epsilon)$ variation of $B_{T}(0)$ with $\theta$ is ignored since it is small and it yjelds no extra information. The poloidal field at the boundary is obtained from the boundary layer solution. From Eq. (28) and the 
scalings, Eq. (4), we obtain,

$$
R_{0} \frac{d\left(B_{p}^{2}\right)_{\psi=0}}{d R}=4 \mu_{0} p(R)
$$

where $p(R)$ is the pressure expressed as a function of major radius in the core. Therefore the boundary data yields the pressure in the core as a function of $R$ (not $\psi$ ). Equations. (62) and (63) can be used in conjunction with the core solution Eq. (11) to give the toroidal flux $\phi(R)$ in the core between $R=R_{0}-a$ and $R$,

$$
\phi=\frac{1}{2 \pi} \int_{R_{0}-a}^{R} d R \sqrt{a^{2}-\left(R_{0}-a\right)^{2}} \sqrt{B_{T}^{2}(0)-\mu_{0} p(R)} .
$$

There is no way to extract $q(\psi)$ from this information. Note that $p(R)$, not $p(\psi)$, is the measurable p profile; Thus, a measurement of $p$ in the core does not add any information to that already deduced from magnetic measurements at the boundary.

Clearly one must measure some other function to deduce $q(\psi)$. Obviously if one could measure $B_{p}(R)$ in the core, Eq. (I1) would yield $q(\psi)$. Measurements of magnetic fields inside the plasma are, however, notoriously difficult. Consider, instead, measuring the pressure along the line $\theta=0$ - thereby measuring $p(R)$ in the core and $p(t)$ in the boundary layer. For monotonic $\mathrm{p}$ we can then define the inverse function, $t(p)$, in the boundary layer. Then, since we know $p(R)$, we express $t=t(p(R))=t(R)$. From Eq. (19) one may deduce $d \psi / d R$ in the core as,

$$
\frac{d \psi}{d R}=2 a^{2}\left(\frac{d t}{d R}\right)\left[\int_{R}^{R_{0}+a} d R^{\prime}\left[1-\left(R^{\prime}-R_{0}\right) / a\right] \mu_{0} \frac{d p}{d R^{\prime}}\right]^{1 / 2}
$$

where $d t / d R$ is the differential of the function $t(R)$. Since the right hand side of Eq. (65) is a known function of $\mathrm{R}$, one may integrate, with the boundary condition $\psi\left(R=R_{0}-a\right)=0$, 
to obtain $\psi(R)$. Thus, the boundary layer equation is used to deduce $\psi(R)$ in the core! The magnetic axis is ignored in this discussion because it only makes a difference in a vanishingly small region.

We can now construct $q(R)$ from Eq. (11),

$$
q(R)=\frac{\sqrt{B_{T}^{2}(0)-\mu_{0} p(R)} \sqrt{a^{2}-\left(R-R_{0}\right)^{2}}}{2 \pi d \psi / d R}
$$

where $d \psi / d R$ is given by Eq. (65). If $q(\psi)$ is reqired one can express $R(\psi)$ by inverting $\psi=$ $\psi(R)$. We have assumed, as before, a knowledge of $B_{T}(0)$ in Eq. (66). This construction can be trivially generalised to shaped tokamaks as follows. Firstly, replace $\sqrt{a^{2}-\left(R-R_{0}\right)^{2}}$ in Eqs. (64) and (65) by $z(R)$, the vertical height of the vacuum vessel at $R$. Secondly, define $R_{0}$ and $a$ in Eq. (65) so that $R=R_{0}+a$ and $R=R_{0}-a$ are the intersections of the conducting wall and the midplane. Note that shaping does not change Eq. (63); therefore the conclusion that $q(\psi)$ cannot be deduced from the boundary data is shape independant.

At very large $\epsilon \bar{\beta}_{p}$ it inay be impossible to measure $p=p(t)$ accurately in the boundary layer. Our construction does, however, demonstrate the theoretical fossibility of deducing the $q$ profile from a measurement of the pressure profile! One possible test of the validity of our expansion is a comparison of the measured $p(R)$ and the one decluced from the measurement of the poloidal field at the boundary. 


\section{References}

${ }^{1}$ J.P. Freidberg, Ideal Magnetohydrodynamics (Plenum, New York, 1987), pp. 154-162.

2J.M. Greene, Plasma Phys. and Cont. Fus. 30, 327 (1988).

${ }^{3}$ J.M. Greene, J.L. Johnson and K.E. Weimer, Phys. Fluids 14, 671 (1971).

${ }^{4}$ V.D. Shafranov, in Reviews of Plasma Physics, Vol.II, ed.M.A. Leontovitch (Consultants Bureau, New York), p.103-151.

${ }^{5}$ J.F. Clarke and D.J. Sigmar, Phys. Rev. Lett. 38, 70 (1977).

${ }^{6}$ M.S. Chance, S.C. Jardin, T.H. Stix, Phys. Rev. Lett. 51, 1963 (1983).

${ }^{7}$ J.W.Connor, R.J.Hastie and J.B.Taylor, Phys. Rev. Lett. 40, 396 (1978).

${ }^{8}$ B.J.Brahms, PhD. Thesis, University of Utrecht, 1986. 


\section{Figures}

FIG. 1. Large aspect ratio geometry.

FIG. 2. Core and boundary layer regions with typical fux surfaces.

FIG. 3. Plot of $\psi_{0}(x)$ for $q=q_{0}=$ constant and $p=p_{0}^{\prime} \psi$ for various values of $\beta_{0}=p_{0}^{\prime} / q_{0}^{2}$. Including a "hole" solution for $\beta_{0}=64$ for which $x_{h}=0$.

FIG. 4. A typical "hole" equilibrium, showing flux surfaces and the "hole".

FIG. 5. Currentless halo surrounding a high beta solution.

FIG. 6. Three regions for the magnetic axis matching. the core region is constrained so that, $t-t_{0} \gg \epsilon^{1 / 6}$ for $\theta \sim O\left(\epsilon^{1 / 3}\right)$ and $t-t_{0} \gg \theta^{1 / 2}$ for $\theta^{1 / 3}$. The magnetic axis Iegion is where $t-t_{0} \sim O\left(\epsilon^{1 / 6}\right)$ and $\theta \sim O\left(\epsilon^{1 / 3}\right)$. The boundary layer region is where $t_{0} \geq t_{0}-t \gg \epsilon^{1 / 6}$ when $\theta \sim O\left(\epsilon^{1 / 3}\right)$ and $t \ll 1$ for $\theta \gg \epsilon^{1 / 3}$.

FIG. 7. Plot of the magnetic axis solution for the "optimum" $s_{0}$. 


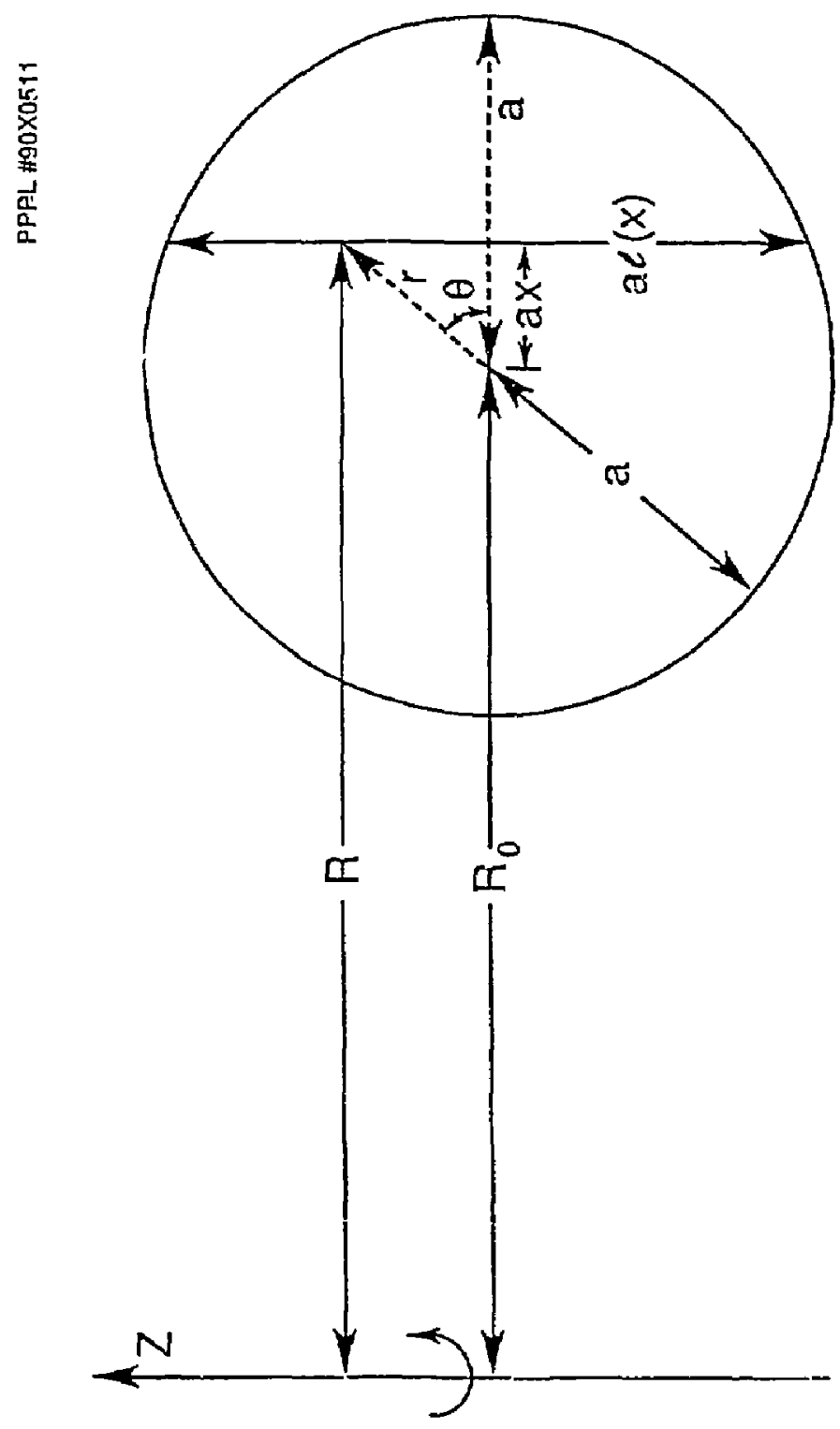

Eig. 1 
PPPL $\# 90 \times 0509$

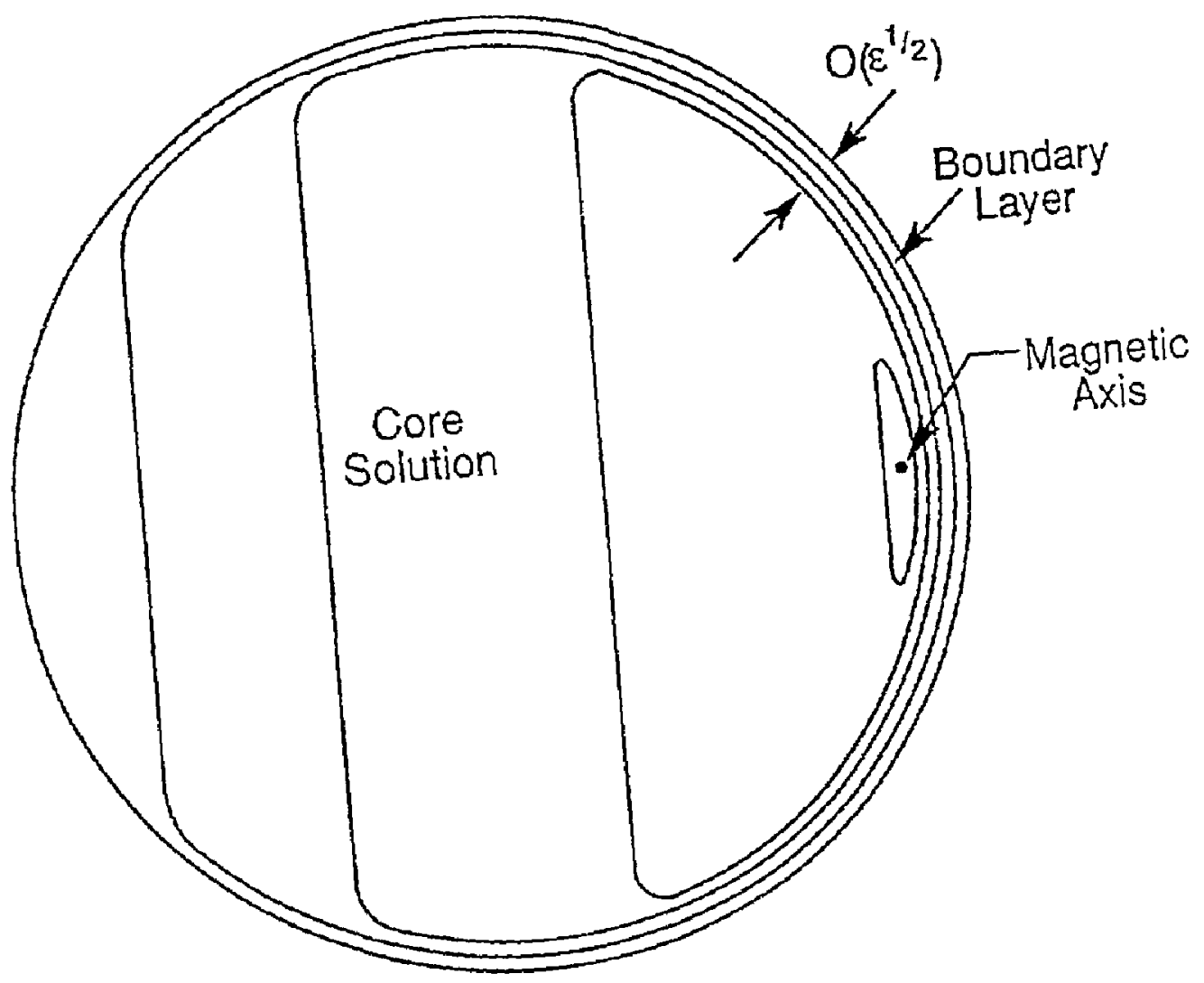

ERg. 2 


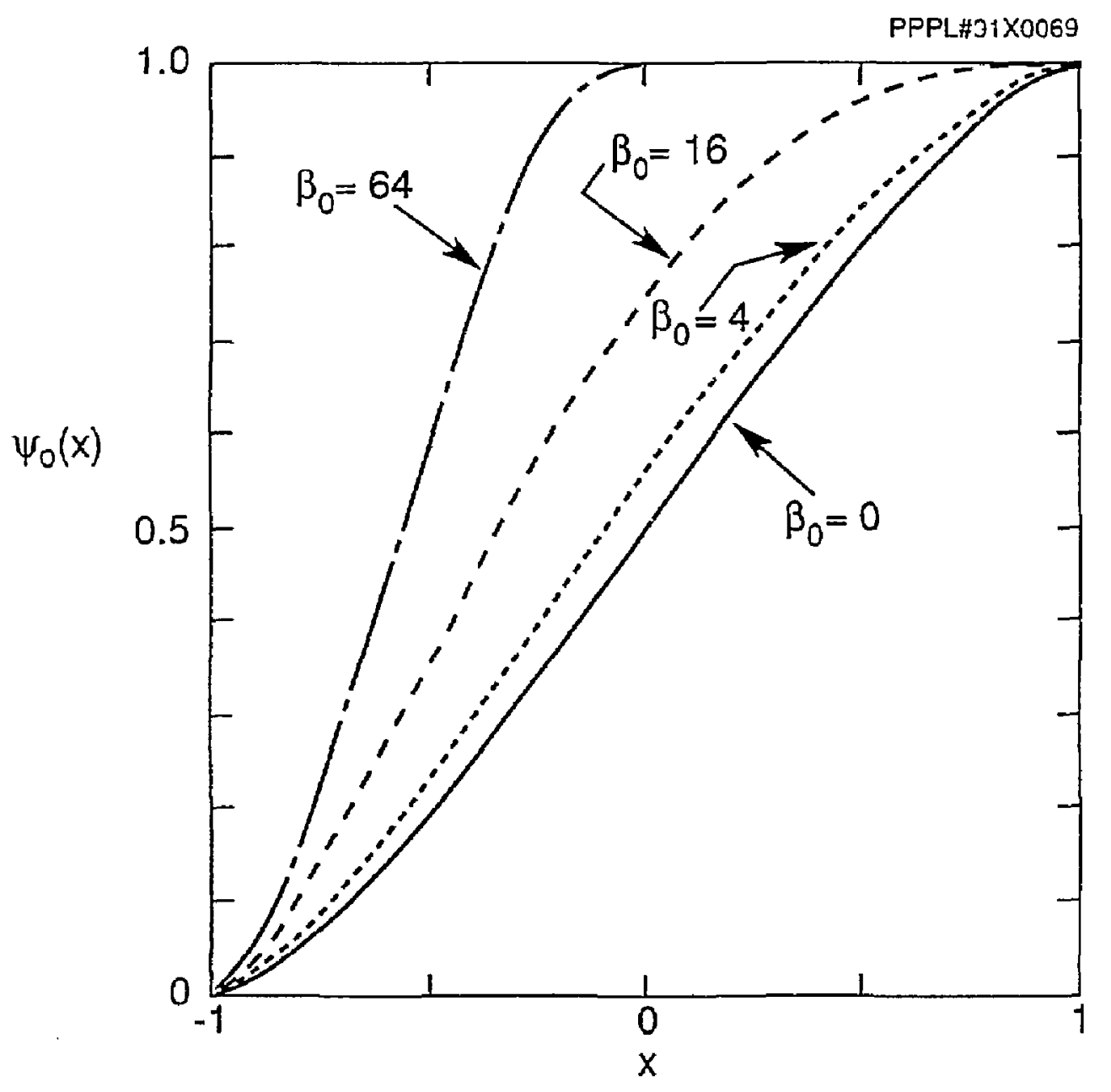

Fig. 3 
PPPL \#90X0509

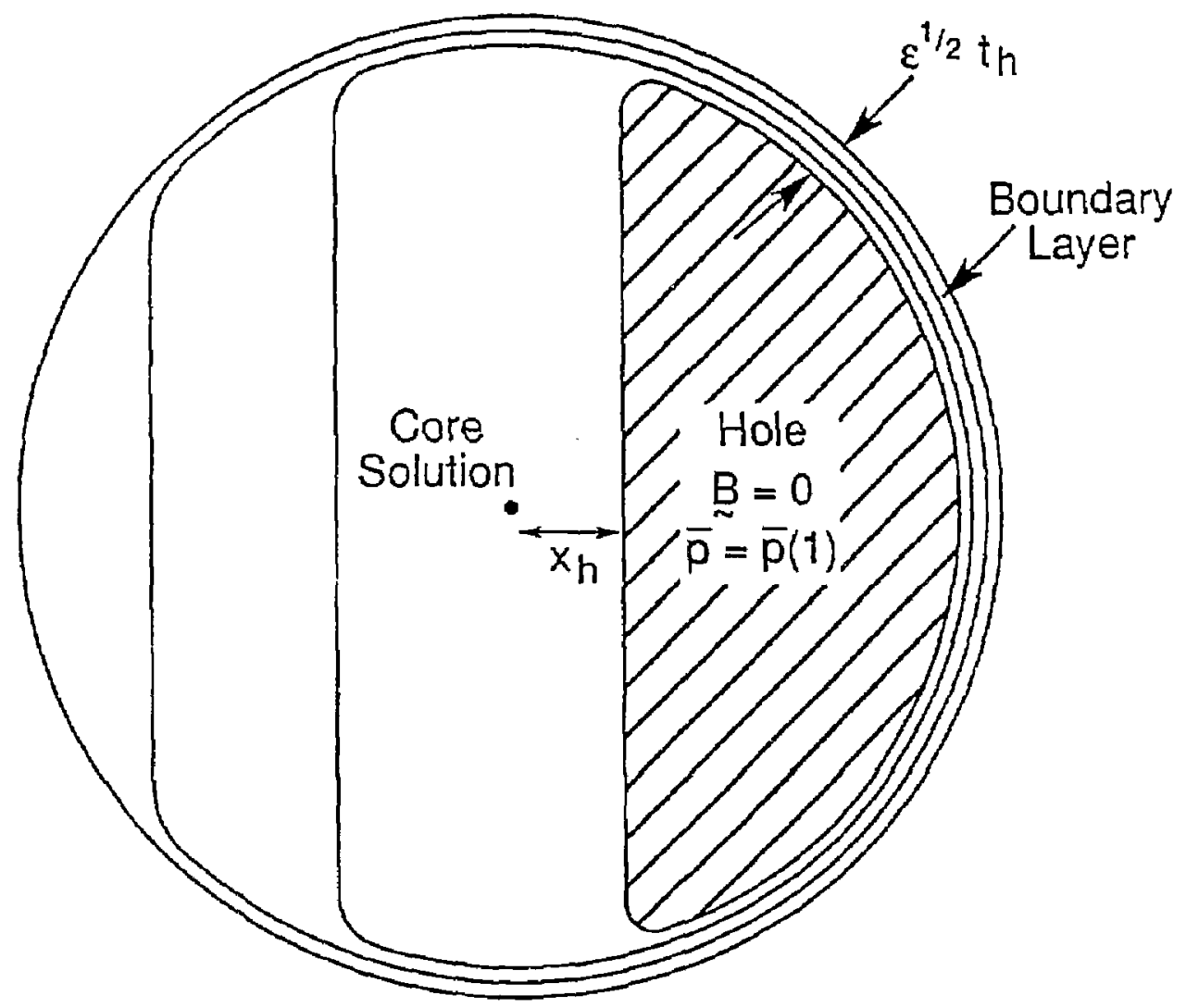

Fig. 4 


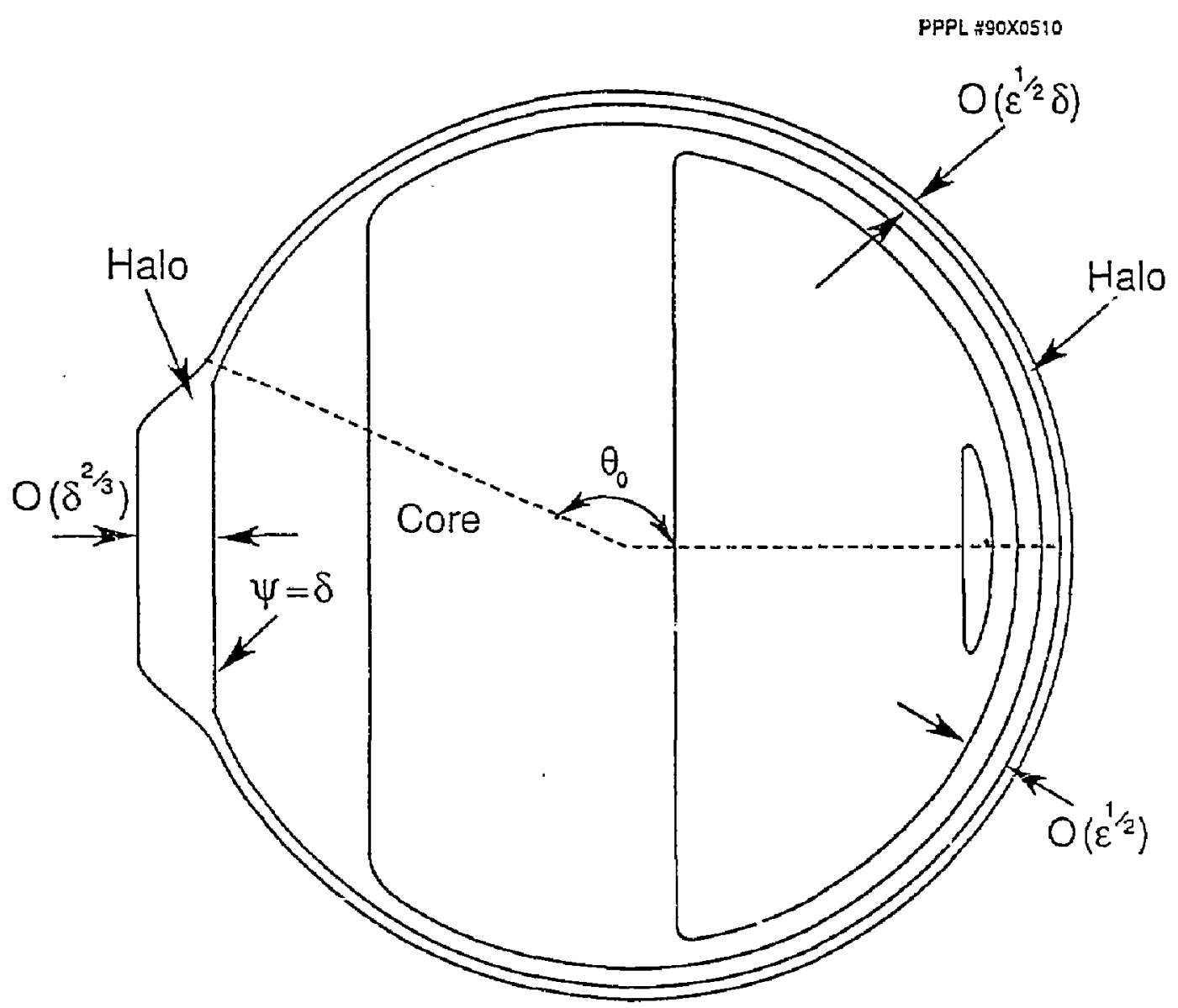

Fig. 5 


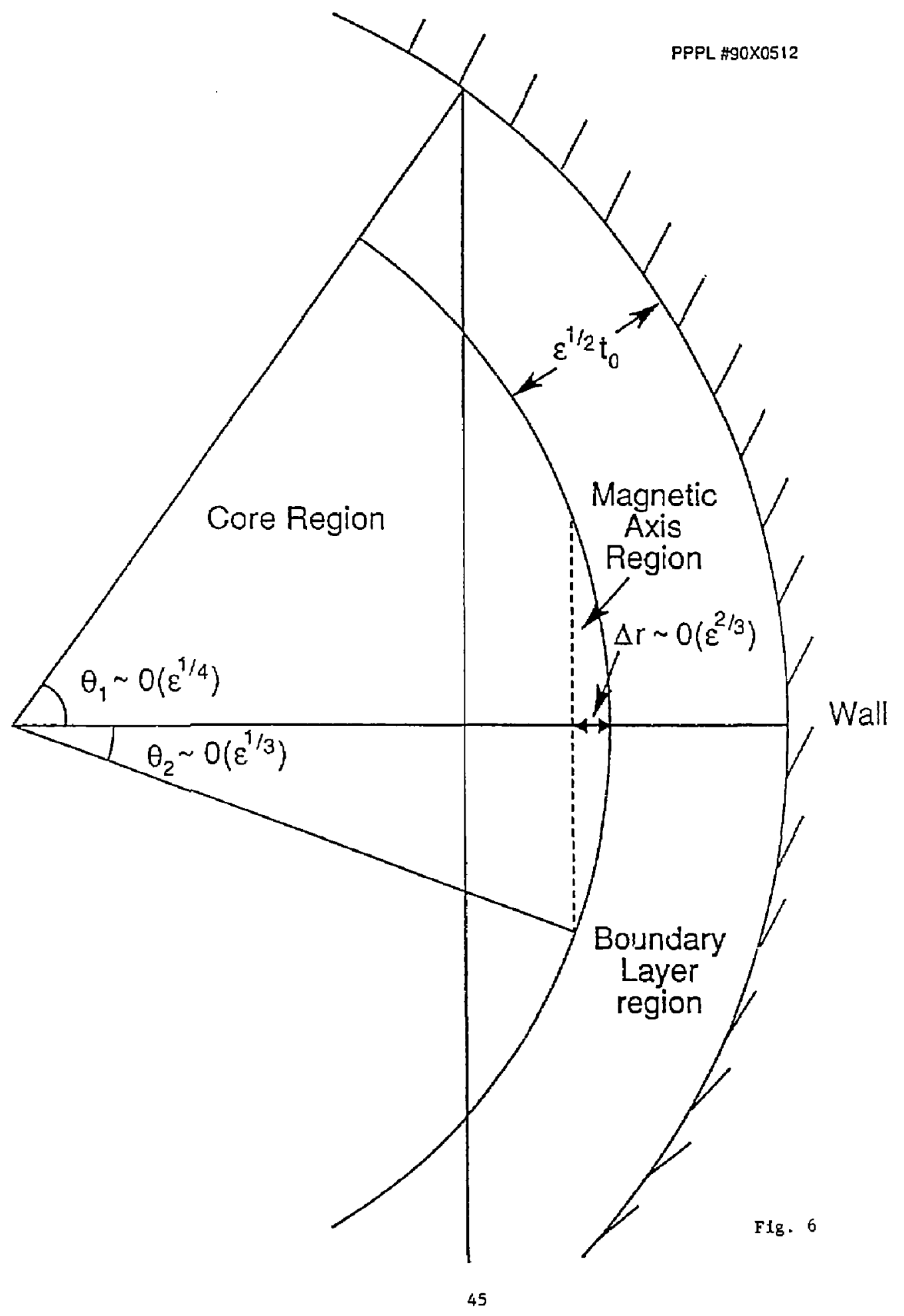




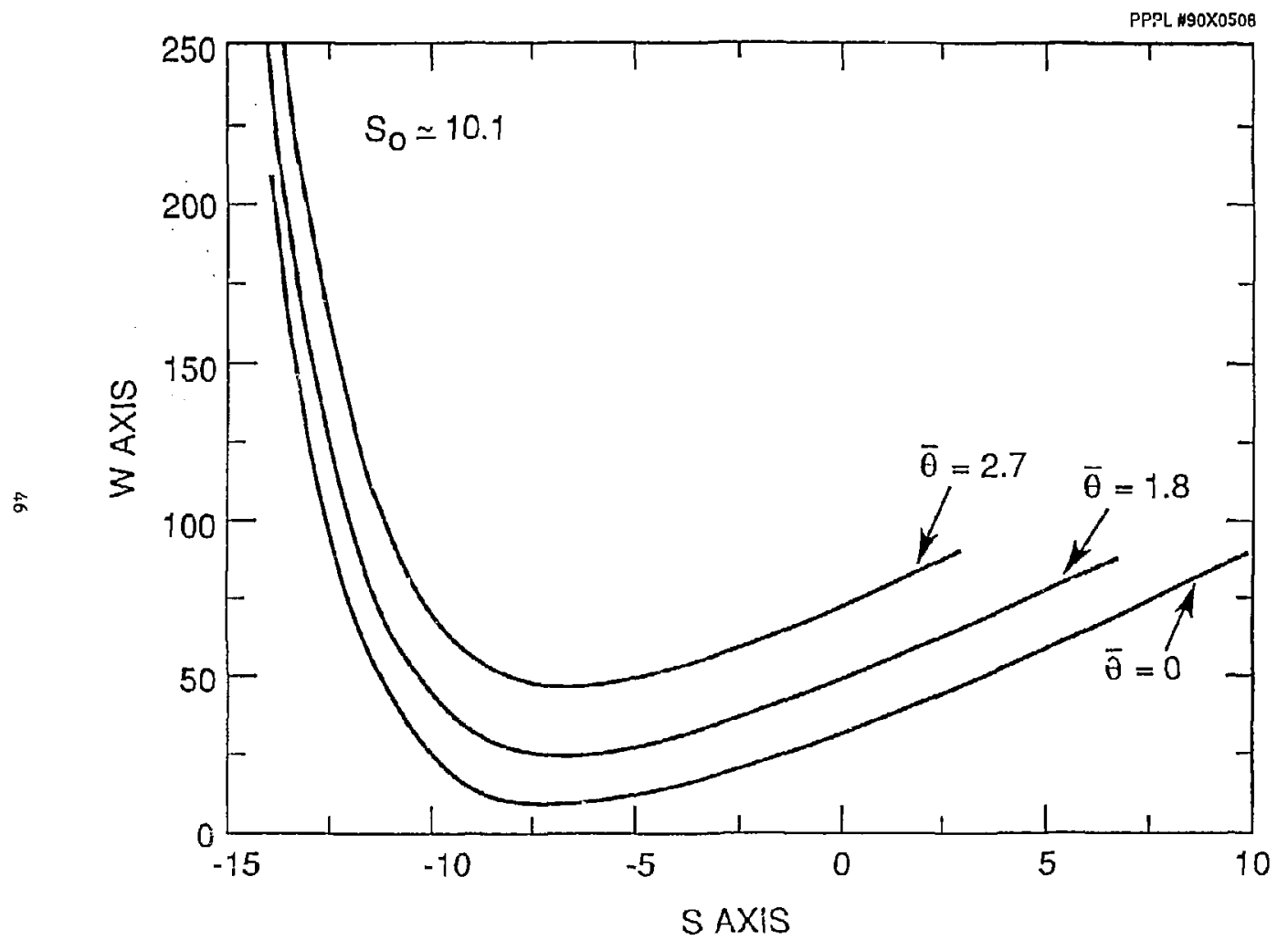

Fig. ? 